\title{
Sedation with dexmedetomidine in elderly patients during dental surgery: a retrospective case series
}

\author{
Mika Seto, Ryosuke Kita, Seiji Kondo \\ Department of Oral and Maxillofacial Surgery, Faculty of Medicine, Fukuoka University, Fukuoka, Japan
}

\begin{abstract}
J Korean Assoc Oral Maxillofac Surg 2019;45:152-157)
Objectives: The number of elderly patients with systemic basal disease requiring invasive dental treatment has increased. Appropriate prediction of surgical invasiveness and combined use of psychosedation are thought to contribute to safe whole-body management. Dexmedetomidine (DEX) exhibits analgesic and anti-anxiolytic properties and causes mild respiratory depression. Studies regarding DEX use in elderly non-intubated patients are scarce. We aimed to use retrospective data to determine an effective dose of DEX to induce adequate sedation in elderly patients undergoing invasive dental surgery under local anesthesia.

Materials and Methods: One hundred two patients aged 70 to 96 years were presumably appropriately controlled with sedation. DEX was administered at an initial loading dose of 2.0 to $3.1 \mu \mathrm{g} / \mathrm{kg} / \mathrm{hr}$ for 10 minutes. We divided the patients into five groups by age and compared their blood pressures and heart rates.

Results: In all five groups, blood pressure decreased suddenly at approximately 15 and 20 minutes after DEX administration. A marked decrease in blood pressure was noted in patients aged 75 to 79 years.

Conclusion: For elderly patients aged 75 years and above, the initial loading dose of DEX needs to be reduced to lower than half that required for young and middle-age adults.
\end{abstract}

Key words: Dexmedetomidine, Sedation, Elderly

[paper submitted 2018. 7. 30 / revised 2018. 9. 29 / accepted 2018. 10. 16]

\section{Introduction}

The number of elderly patients with systemic basal disease requiring invasive dental treatment increases yearly. Elderly patients are often referred to the oral surgery department at our hospital because perioperative whole-body management can be performed safely. For example, it is easy to coordinate with the relevant specialties, the patient can be treated as an inpatient, and an emergency response can be performed, if necessary.

Dexmedetomidine (DEX) $)^{1}$ exhibits analgesic and anxio-

\section{Mika Seto}

Department of Oral and Maxillofacial Surgery, Faculty of Medicine, Fukuoka University, 7-45-1 Nanakuma, Jonan-ku, Fukuoka 814-0180, Japan

TEL: +81-92-801-1011 FAX: +81-92-801-1044

E-mail:miichan@fukuoka-u.ac.jp

ORCID: https://orcid.org/0000-0002-7548-7613

(a) This is an open-access article distributed under the terms of the Creative Commons Attribution Non-Commercial License (http://creativecommons.org/ licenses/by-nc/4.0/), which permits unrestricted non-commercial use, distribution, and reproduction in any medium, provided the original work is properly cited.

Copyright (C) 2019 The Korean Association of Oral and Maxillofacial Surgeons. All rights reserved. lytic properties and causes mild respiratory depression. In Japan, DEX was recently cleared for use in non-intubated patients requiring sedation. Regarding the dose of DEX for elderly patients, the manufacturer's information only advises that the clinician should "take account of the deceleration of dosing speed and dose it in a careful manner while observing the physical status of the patient," and a clear standard is not available. Studies regarding DEX use in elderly, nonintubated patients are scarce. We have observed the onset of unpredictable, sudden hypotension in the earliest phase of DEX sedation but found that good sedative management was possible by reducing the dosage.

In this study, we aimed to determine an effective dose of DEX to induce adequate sedation in elderly patients undergoing invasive dental surgery under local anesthesia using retrospective data.

\section{Materials and Methods}

After the Ethics Board of Fukuoka University Hospital (Fukuoka, Japan) granted an exemption (No. 15-10-12) for 
this study, the case records of patients aged 70 years and older who underwent elective invasive dental surgery under local anesthesia using DEX sedation from September 2013 through July 2016 were retrospectively extracted. We included the medical records of 124 patients (age range, 70-96 years). The American Society of Anesthesiologists physical status (ASA PS) classification system is a widely used sixpoint scale to describe preoperative physical status. All patients in this study had at least one baseline disease for which the ASA PS classification ranged from Class 2 to 3 . The patients were hospitalized on the day before surgery. On the day of treatment, the patient was admitted to an operating room where their vital signs, including noninvasive blood pressure, electrocardiography, heart rate, and peripheral oxygen saturation, were continuously monitored and an intravenous line was inserted. Oxygen was administered at $3 \mathrm{~L} / \mathrm{min}$ through a nasal cannula. The DEX dosage $(3 \mu \mathrm{g} / \mathrm{kg} / \mathrm{hr})$ was determined using body weight according to the manufacturer's guidelines. When the weight of the patient was higher than the standard weight, a dose based on the standard weight was used, and the initial loading dose was calculated by dividing the amount used by the actual body weight. When the patient was petite, we reduced the dose of DEX using low body weight as a guideline. Sedation depth was assessed using the
Observer's Assessment of Alertness/Sedation (OAA/S) scale. OAA/S scores (1 to 5 ) and vital signs were recorded every 5 minutes. For the analysis, we examined patient characteristics, DEX dosage, type of operation, hemodynamics, and respiratory variables. Detailed examinations were conducted of patients who suffered complications that required perioperative rescue medication and of patients dissatisfied with their sedation. An additional investigation was then performed to assess DEX doses by age on the assumption that the remaining patients had been adequately controlled via sedation.

\section{Results}

Four patients were administered emergency drugs due to complications, and these cases are detailed in Table 1. Two patients exhibited severe bradycardia and required the administration of atropine sulfate $(0.5 \mathrm{mg})$. Four patients became hypotensive and required the administration of a vasopressor agent during sedation. Two patients were dissatisfied with the sedation; they complained that they felt uncomfortable after surgery. We administered a maintenance dose of DEX for only 16 patients. One hundred and two patients aged 70 to 96 years were presumably appropriately controlled with only a single loading dose of DEX (Table 2). The number of

Table 1. Complications occurring during invasive oral surgery with dexmedetomidine (DEX) sedation

\begin{tabular}{cccclccc}
\hline $\begin{array}{c}\text { Patient } \\
\text { No. }\end{array}$ & $\begin{array}{c}\text { Age } \\
(\mathrm{yr})\end{array}$ & Sex & $\begin{array}{c}\text { DEX loading } \\
\text { dose }(\mu \mathrm{g} / \mathrm{kg} / \mathrm{hr})\end{array}$ & Complication & $\begin{array}{c}\text { BP } \\
(\mathrm{mmHg})\end{array}$ & $\begin{array}{c}\text { HR } \\
(\mathrm{bpm})\end{array}$ & Emergency drugs administered \\
\hline 1 & 73 & $\mathrm{M}$ & 3.0 & Persistent hypotension, bradycardia & $78 / 52$ & 40 & Ephedrine 8 mg IV, atropine sulfate 0.5 mg \\
2 & 70 & $\mathrm{~F}$ & 3.0 & Persistent hypotension, bradycardia & $80 / 49$ & 43 & Ephedrine 4 mg IV, atropine sulfate 0.5 mg \\
3 & 85 & $\mathrm{~F}$ & 5.0 & Persistent hypotension & $80 / 48$ & 62 & Phenylephrine 0.2 mg IV \\
4 & 86 & $\mathrm{~F}$ & 4.5 & Persistent hypotension & $78 / 59$ & 68 & Phenylephrine 0.2 mg IV \\
\hline
\end{tabular}

(M: male, F: female, BP: blood pressure, HR: heart rate, IV: intravenous administration)

Mika Seto et al: Sedation with dexmedetomidine in elderly patients during dental surgery: a retrospective case series. J Korean Assoc Oral Maxillofac Surg 2019

Table 2. Patient background

\begin{tabular}{|c|c|c|c|c|c|}
\hline & \multicolumn{5}{|c|}{ Age group (yr) } \\
\hline & $70-74$ & $75-79$ & $80-84$ & $85-89$ & $90-96$ \\
\hline No. of patients $(\mathrm{M} / \mathrm{F})$ & $20(10 / 10)$ & $32(21 / 11)$ & $32(15 / 17)$ & $11(4 / 7)$ & $7(2 / 5)$ \\
\hline Height (cm) & $155.8 \pm 8.8$ & $155.2 \pm 6.8$ & $157.6 \pm 10.1$ & $145.8 \pm 12.6$ & $155.8 \pm 8.8$ \\
\hline Weight (kg) & $58.1 \pm 8.2$ & $58.1 \pm 9.5$ & $50.5 \pm 7.4$ & $47.9 \pm 4.9$ & $48.7 \pm 14.9$ \\
\hline \multicolumn{6}{|l|}{ Baseline disease (overlapping) } \\
\hline Congestive heart failure & 5 & 12 & 5 & 5 & 2 \\
\hline Coronary artery disease & 10 & 23 & 19 & 8 & 3 \\
\hline Hypertension & 19 & 30 & 28 & 11 & 7 \\
\hline Diabetes & 3 & 15 & 10 & 5 & 0 \\
\hline Liver disease & 2 & 10 & 10 & 2 & 0 \\
\hline Kidney dysfunction $($ GFR <60) & 12 & 29 & 31 & 9 & 5 \\
\hline Any comorbidity & 0 & 0 & 0 & 0 & 0 \\
\hline
\end{tabular}

(M: male, F: female, GFR: glomerular filtration rate)

Values are presented as number only or mean \pm standard deviation.

Mika Seto et al: Sedation with dexmedetomidine in elderly patients during dental surgery: a retrospective case series. JKorean Assoc Oral Maxillofac Surg 2019 
patients aged 85 years or older was 18 . The sedation depth of these patients was such that they reacted when spoken to but otherwise kept breathing as if they were asleep. The OAA/ $\mathrm{S}$ scores for all patients during surgery ranged from 4 to 5 . Most candidates had cardiovascular diseases or hypertension as a systemic underlying disease and many had complications from multiple underlying diseases. The renal function of most patients older than 75 years of age was mildly decreased (glomerular filtration rate $<60$ ). We divided the patients into 5 groups by age in increments of 5 years and compared their mean blood pressures and heart rates. These patients' perioperative general conditions were stable, and their primary diseases or other complications were not exacerbated. The mean blood pressure in all groups was higher just before treatment than at the time of hospital admission. (Fig. 1) DEX was administered at an initial loading dose of 2.0 to $3.1 \mu \mathrm{g} / \mathrm{kg} / \mathrm{hr}$ for 10 minutes.(Table 3 ) The mean loading dose for DEX decreased by 0.3 to $0.4 \mu \mathrm{g} / \mathrm{kg} / \mathrm{hr}$ with each increase in age group; however, there was no change in the loading dose between the 80- to 84-year group and the 85to 89-year group. All patients had a slower heart rate (Fig. 2), lower mean blood pressure (Fig. 1), and cooperated more readily compared to their preoperative status. There were no notable changes in blood pressure in any group for the first 10 minutes after commencing DEX administration, but blood pressure decreased suddenly at approximately 15 to $20 \mathrm{~min}$ utes after DEX administration. The mean blood pressure was lowest in those aged 75 to 79 years; moreover, the decrease in blood pressure after DEX administration was substantial. Heart rate also tended to decrease starting after 10 minutes of DEX administration. No serious adverse effects were recorded.

\section{Discussion}

As the extents of anxiety and potential complications
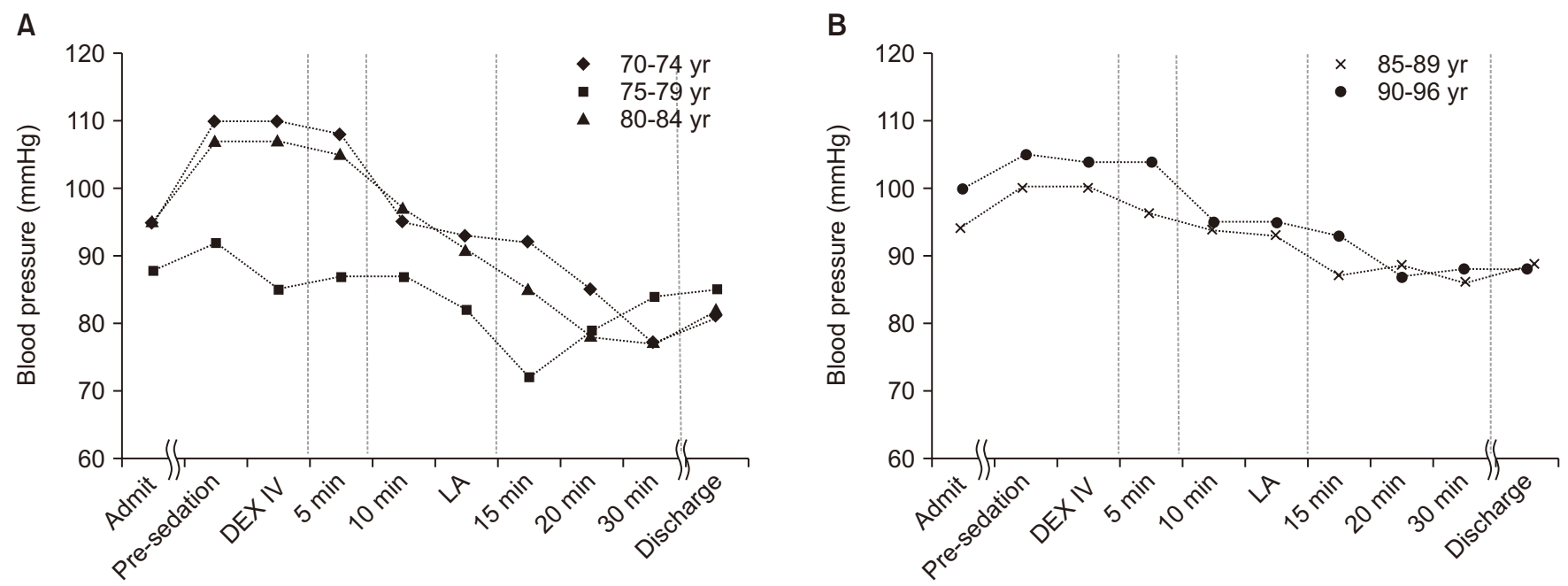

Fig. 1. A, B. Transitional changes in mean blood pressure. (Admit: admission time, Pre-sedation: walking into the operating room, DEX IV: immediately before administration of dexmedetomidine, 5 min: 5 minutes after the administration of dexmedetomidine, 10 min: 10 minutes after the administration of dexmedetomidine, LA: immediately after infiltration anesthesia, 15 min: 15 minutes after the administration of dexmedetomidine, 20 min: 20 minutes after the administration of dexmedetomidine, 30 min: 30 minutes after the administration of dexmedetomidine, Discharge: immediately before returning to the ward)

Mika Seto et al: Sedation with dexmedetomidine in elderly patients during dental surgery: a retrospective case series. J Korean Assoc Oral Maxillofac Surg 2019

Table 3. Operation and dosage amount of dexmedetomidine (DEX)

\begin{tabular}{|c|c|c|c|c|c|}
\hline & \multicolumn{5}{|c|}{ Age group (yr) } \\
\hline & $70-74$ & $75-79$ & $80-84$ & $85-89$ & $90-96$ \\
\hline Duration of surgery (min) & $42.5 \pm 10.4$ & $49.9 \pm 18.2$ & $48.5 \pm 7.8$ & $34.2 \pm 6.3$ & $28.5 \pm 17.1$ \\
\hline DEX loading dose $(\mu \mathrm{g} / \mathrm{kg} / \mathrm{hr})$ & $3.1 \pm 1.8$ & $2.7 \pm 1.3$ & $2.4 \pm 0.6$ & $2.4 \pm 0.7$ & $2.0 \pm 0.3$ \\
\hline \multicolumn{6}{|l|}{ Operative method (overlapping) } \\
\hline Teeth extraction & 19 & 32 & 32 & 9 & 5 \\
\hline Cystectomy & 8 & 3 & 2 & 0 & 1 \\
\hline Other & 1 & 0 & 0 & 2 & 2 \\
\hline
\end{tabular}

Values are presented as number only or mean \pm standard deviation.

Mika Seto et al: Sedation with dexmedetomidine in elderly patients during dental surgery: a retrospective case series. J Korean Assoc Oral Maxillofac Surg 2019 
A

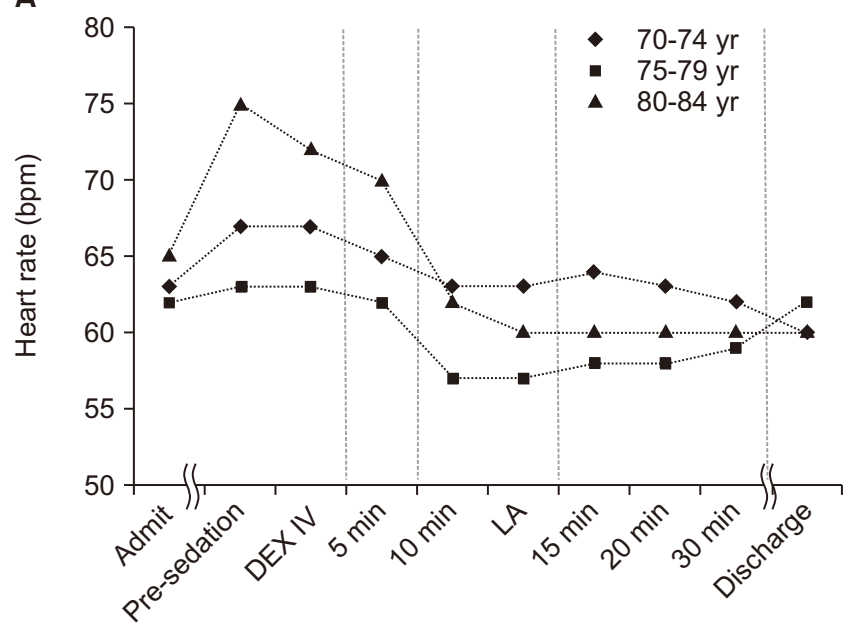

B

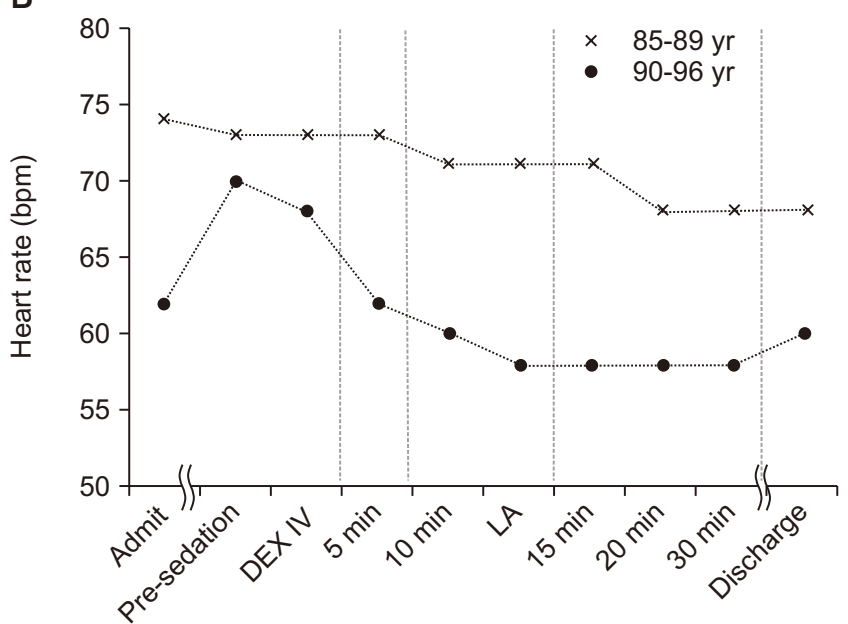

Fig. 2. A, B. Transitional changes in heart rate. Refer to Fig. 1 for the definition of the abbreviations.

Mika Seto et al: Sedation with dexmedetomidine in elderly patients during dental surgery: a retrospective case series. J Korean Assoc Oral Maxillofac Surg 2019

among elderly patients vary, it is essential to understand the physical status of the patient just before surgery in invasive treatment and to manage it during treatment by monitoring the patient. Moreover, an appropriate prediction of surgical invasiveness and the combined use of psychosedation are thought to contribute to the safe control of the whole body. In our clinical practice, we treated a patient with a history of hypertension whose preoperative blood pressure control was judged as good but whose blood pressure and heart rate increased just before surgery, which was thought to be caused by anxiety. In fact, the blood pressure of this specific patient was higher just before treatment than at the time of hospital admission. To perform safe treatments, it is necessary to provide intravenous sedation based on the reduction in function and reserve capacity of the organs due to aging.

$\mathrm{DEX}^{1}$, an agonist of $\alpha_{2}$-adrenergic receptors in certain parts of the brain, is commonly used as a sedative. The most frequent adverse reactions to DEX are hypotension and bradycardia $^{2,3}$. Elderly patients have several common physiological characteristics including decreased cardiac and respiratory function, reductions in drug clearance, diseases related to aging, other chronic diseases, and hypertension. Many reports indicate that sedation can help stabilize hemodynamics in elderly patients during invasive treatments ${ }^{4-6}$. Appropriate sedation contributes to a reduction in the patient's psychological and physiological stress. Difficulties associated with sedation in the elderly ${ }^{7}$ include airway obstruction, respiratory depression, coughing due to inhaling secretions, and hypotension. Midazolam and propofol are the most commonly used sedatives. Midazolam ${ }^{8}$ induces dose-related settling of the tongue root and respiratory depression, and propofol $^{4}$ produces inhibitory effects on the heart and cardiovascular system in addition to causing respiratory depression. On the other hand, $\mathrm{DEX}^{2,3}$ has a wide range of pharmacological effects including sedative, analgesic, and anxiolytic properties, as well as hemodynamic stabilization effects that relieve sympathetic hyperactivity due to stress. In addition, DEX has cardioprotective effects, induces mild respiratory depression, and patients administered DEX were reported to have "maintained orientation to the environment." MacLaren et al. ${ }^{9}$ reported that DEX may be associated with less delirium compared with other sedatives. Kunisawa et al. ${ }^{10}$ reported that DEX was safely used for sedation in a very elderly patient. From these reports and our results, we believe that DEX contributes to the safe perioperative management of elderly patients, and we have started to use DEX as a sedative. We administered an initial loading dose of $6 \mu \mathrm{g} / \mathrm{kg} / \mathrm{hr}$ to all patients when we began the use of DEX for sedation. No complications were noted in patients aged 20 to 40 years. However, in patients over 65 years of age, episodes of sudden bradycardia and hypotension occurred, necessitating the administration of emergency medications. To ensure the safe administration of drugs in the elderly, an initial dose smaller than that used for other adults should be utilized because their liver and kidney function have likely declined ${ }^{11}$. Therefore, the initial loading dose of DEX was reduced to $3 \mu \mathrm{g} / \mathrm{kg} / \mathrm{hr}$ or less in elderly patients over 70 years old.

However, even at this lower dose, the decrease in blood pressure was massive in patients aged 75 to 79 years in comparison to patients in the other age groups. Therefore, we 
suggest a dose of less than $2.7 \mu \mathrm{g} / \mathrm{kg} / \mathrm{hr}$ for those aged 75 to 79 years. This fact may be useful when designing prospective studies to determine the initial loading dose of DEX in the elderly.

We have observed cases in which local anesthesia rapidly increased the blood pressure or heart rate during sedation with midazolam. During sedation with DEX, however, there were no significant changes in hemodynamics even after administration of local anesthesia. This is likely because of the strong analgesic effect of DEX.

The sedation depth achieved by midazolam administration is adjusted according to blood pressure and sedation score (e.g., the Ramsey Sedation Score or OAA/S). In our cases involving DEX administration, blood pressure did not change just after administration commenced, but it began to decrease 15 to 20 minutes later. Therefore the dosage could not be adjusted using blood pressure as an indicator. In addition, the sedation score could not be used as an indicator because the patient was easily awakened by soft stimulation.

Ok et al. ${ }^{12}$ reported that a loading dose of $6 \mu \mathrm{g} / \mathrm{kg} / \mathrm{hr}$ of DEX was sufficient for spinal anesthesia surgery lasting less than 60 minutes. The half-life of DEX is increased according to the severity of liver dysfunction. Its metabolite is mainly excreted in the urine and could accumulate in patients with renal dysfunction. In addition, the liver and renal function values for our patients were lower than normal. Therefore our results suggest that it is possible to perform dental surgery approximately 60 minutes in duration in patients older than 70 years with an initial loading dose of DEX less than $3 \mu \mathrm{g} /$ $\mathrm{kg} / \mathrm{hr}$ for 10 minutes.

Kim et al. ${ }^{13}$ reported that the single dose of DEX required for sedation was $21 \%$ lower in older men (65-78 years old) compared to younger men (45-64 years old) who underwent transurethral resection of the prostate with spinal anesthesia. Our results show that among patients who underwent dental surgery under local anesthesia, the single dose of DEX required for sedation was $22.5 \%$ lower in the group aged 80 years and older compared to the group aged 70 to 74 years. While there can be great individual variability in hemodynamic effects, an appropriate dose of DEX effectively enables the stabilization of hemodynamics in elderly patients.

\section{Conclusion}

To sedate elderly patients aged 70 years or older, we recommend that the initial DEX loading dose should be $3 \mu \mathrm{g} /$ $\mathrm{kg} / \mathrm{hr}$ or less. Moreover, as the age increases, it is necessary to further reduce the initial loading dose from the dose calculated according to body weight.

\section{ORCID}

Mika Seto, https://orcid.org/0000-0002-7548-7613
Ryosuke Kita, https://orcid.org/0000-0003-4526-5275
Seiji Kondo, https://orcid.org/0000-0001-9341-5069

\section{Authors' Contributions}

M.S. participated in the study design, data collection and wrote the manuscript. R.K. participated in data collection. S.K. participated in the study design and coordination and helped to draft the manuscript. All authors read and approved the final manuscript.

\section{Ethics Approval and Consent to Participate}

This study was approved by the Ethics Board of Fukuoka University Hospital (Fukuoka, Japan) (approval No. 15-1012) and the written informed consent was obtained from all patients.

\section{Conflict of Interest}

No potential conflict of interest relevant to this article was reported.

\section{References}

1. Cormack JR, Orme RM, Costello TG. The role of alpha2-agonists in neurosurgery. J Clin Neurosci 2005;12:375-8.

2. Ebert TJ, Hall JE, Barney JA, Uhrich TD, Colinco MD. The effects of increasing plasma concentrations of dexmedetomidine in humans. Anesthesiology 2000;93:382-94.

3. Constantin JM, Momon A, Mantz J, Payen JF, De Jonghe B, Perbet $\mathrm{S}$, et al. Efficacy and safety of sedation with dexmedetomidine in critical care patients: a meta-analysis of randomized controlled trials. Anaesth Crit Care Pain Med 2016;35:7-15.

4. Cillo JE Jr, Finn R. Hemodynamics in elderly coronary artery disease patients undergoing propofol sedation. J Oral Maxillofac Surg 2006;64:1338-42.

5. Taguchi T, Fukuda K, Sekine H, Kakizawa T. Intravenous sedation and hemodynamic changes during dental implant surgery. Int $\mathrm{J}$ Oral Maxillofac Implants 2011;26:1303-8.

6. Hegde S, Padmanabha S, Bhat GK. A comparison of hemodynamic responses and patient comfort in catract surgery with and without midazolam as sedation. IOSR J Dent Med Sci 2014;13:23-7.

7. Silva-Jr JM, Katayama HT, Nogueira FAM, Moura TB, Alves TL, de Oliveira BW. Comparison of dexmedetomidine and benzodiazepine for intraoperative sedation in elderly patients: a randomized clinical trial. Reg Anesth Pain Med 2019;44:319-24.

8. Kanto JH. Midazolam: the first water-soluble benzodiazepine. 
Pharmacology, pharmacokinetics and efficacy in insomnia and anesthesia. Pharmacotherapy 1985;5:138-55.

9. MacLaren R, Preslaski CR, Mueller SW, Kiser TH, Fish DN, Lavelle JC, et al. A randomized, double-blind pilot study of dexmedetomidine versus midazolam for intensive care unit sedation: patient recall of their experiences and short-term psychological outcomes. J Intensive Care Med 2015;30:167-75.

10. Kunisawa T, Hanada S, Kurosawa A, Suzuki A, Takahata O, Iwasaki H. Dexmedetomidine was safely used for sedation during spinal anesthesia in a very elderly patient. J Anesth 2010;24:938-41.

11. Shah RR. Drug development and use in the elderly: search for the right dose and dosing regimen (Parts I and II). Br J Clin Pharmacol 2004;58:452-69.

12. Ok HG, Baek SH, Baik SW, Kim HK, Shin SW, Kim KH. Optimal dose of dexmedetomidine for sedation during spinal anesthesia. Korean J Anesthesiol 2013;64:426-31.

13. Kim J, Kim WO, Kim HB, Kil HK. Adequate sedation with singledose dexmedetomidine in patients undergoing transurethral resection of the prostate with spinal anaesthesia: a dose-response study by age group. BMC Anesthesiol 2015;15:17.

How to cite this article: Seto M, Kita R, Kondo S. Sedation with dexmedetomidine in elderly patients during dental surgery: a retrospective case series. J Korean Assoc Oral Maxillofac Surg 2019;45:152-7. https://doi.org/10.5125/jkaoms.2019.45.3.152 